\title{
O CLIMA DE ONDAS NA COSTA SUL DE ARRAIAL DO CABO, RJ: DISTRIBUIÇÃO DE PARÂMETROS DE ONDA
}

\author{
PEREIRA, J. ${ }^{1}$; CANDELLA, R.N. ${ }^{2}$ \& J.L.B. CARVALHO ${ }^{3}$ \\ 1 - Bolsista Art. 170/UNIVALI - Laboratório de Oceanografia Física. e-mail: \\ janini@mailcity.com \\ 2 - Instituto de Estudos do Mar Almirante Paulo Moreira (IEAPM) - Marinha do Brasil \\ 3 - Universidade do Vale do Itajaí (UNIVALI) - Laboratório de Oceanografia Física
}

\begin{abstract}
RESUMO
A Marinha do Brasil operou um um ondógrafo direcional WAVERIDER DATAWELL ao largo da costa sul de Arraial do Cabo, RJ, sobre uma lâmina d'água de 82 metros, entre agosto de 1988 e outubro de 1999. Os resultados do processamento preliminar das informações de onda direcional são apresentados. As séries temporais de curto termo foram consistidas e então submetidas a rotinas de cálculo do espectro direcional. As séries de parâmetros amostrais como altura significativa, período de pico, direção e espalhamento no período de pico foram sintetizadas na forma de histogramas e diagramas de ocorrência conjunta. Os resultados mostraram que a maior parte da energia provêm de ondas vindas de SSE a SSO, com períodos variando entre 11 e 13 segundos. Um segundo pico de energia vem de ESE a SSE com períodos predominando entre 7 e 9 segundos. O espalhamento das ondas vindas de $\mathrm{S}$, geradas mais distante, foi ligeiramente maior do que as de $\mathrm{SE}$. Isto pode estar relacionado com uma maior variabilidade espaço-temporal dos ventos nas respectivas zonas de geração.
\end{abstract}

Palavras chave: Clima de ondas, Espectro direcional de ondas, Litoral do Rio de Janeiro

\section{WAVE CLIMATE AT THE SOUTH COAST OF ARRAIAL DO CABO, RIO DE JANEIRO, BRAZIL: DISTRIBUTION OF WAVE PARAMETERS}

\begin{abstract}
The Brazilian Navy operated a directional wave meter WAVERIDER DATAWELL off shore of the Arraial do Cabo South coast, Rio de Janeiro, Brazil, at 82-m water column, from august, 1998 to October, 1999. The preliminary results of the directional wave processing are presented. The short-term time series were consisted and then submitted to directional spectra procedures. Time hystories of wave parameters, such as significant height, period peak, mean direction and spreading at the peak period were synthesized on histograms and joint occurrence diagrams. The results show that most of the energy comes from SSE to SSW, with periods varying from 11 to 13 seconds. A second peak of energy comes from ESE to SSE with periods varying from 7 to 9 seconds. The spreading of the southerly waves, farthermost generated, were slightly greater than of the SE ones. It can be, possibly, related to a bigger space-time variability of the winds in their respective generation zone.
\end{abstract}

Keywords: Wave climate, Directional wave spectra, Rio de Janeiro coast.

\section{INTRODUÇÃO}

Ondas de gravidade geradas pelo vento são as principais responsáveis pelo transporte de sedimentos em praias oceânicas. O conhecimento das alturas, dos períodos e das direções das ondas no início da zona de arrebentação, bem como as características geomorfológicas da praia, são fundamentais ao conhecimento da evolução morfológica das mesmas, permitindo, por exemplo, prever respostas a possíveis ações antrópicas. 
A determinação das características das ondas no início da zona de arrebentação é usualmente efetuada através de modelos de refração-difração que utilizam como condições de contorno informações de ondas medidas em pontos com lâmina d'água superiores. O conhecimento da variação temporal e da estatística dos parâmetros de onda, a compreensão dos eventos meteorológicos que geraram os respectivos estados de mar e a distribuição de energia por faixas de período em cenários freqüentes compõem o estudo do clima de ondas para uma determinada região. Quanto melhor for o conhecimento do clima de ondas melhor será a compreensão dos processos morfodinâmicos que ocorrem na praia.

Estudos de clima de ondas na costa brasileira efetuados com ondógrafos direcionais são ainda raros. O clima de ondas da Bacia de Campos foi bem caracterizado utilizando informações obtidas pela PETROBRAS em águas profundas (Violante Carvalho et al, 1995; Violante Carvalho,1998; Seixas, 1997). No Rio Grande do Sul, Strauch \& Schimidt (1998), apresenta resultados de um ano de monitoramento em águas rasas. Paradoxalmente, a região sudeste brasileira, apesar da importância econômica do setor naval e de dispor da maioria das instituições e recursos financeiros voltados à pesquisa no Brasil, ainda é deficiente nesta área.

Entre agosto de 1998 e outubro de 1999, com o objetivo de caracterizar o clima de ondas na região sul de Arraial do Cabo, RJ, o Instituto de Estudos do Mar Almirante Paulo Moreira, operou um ondógrafo direcional WAVERIDER. A campanha foi bem sucedida obtendo uma taxa de recuperação dos dados superior a $98 \%$.

O presente trabalho propõe-se a apresentar os resultados iniciais do processamento dos dados obtidos pelo ondógrafo. Resultados estes que irão compor a determinação do clima de ondas da região, contribuindo assim com o preenchimento da lacuna existente de informações de ondas com resolução direcional na costa sudeste brasileira.

A Figura 1 mostra a localização do fundeio do ondógrafo. O mesmo foi instalado às coordenadas $23^{\circ} 01^{\prime} \mathrm{S}$ e $042^{\circ} 03^{\prime} \mathrm{O}$, sobre uma

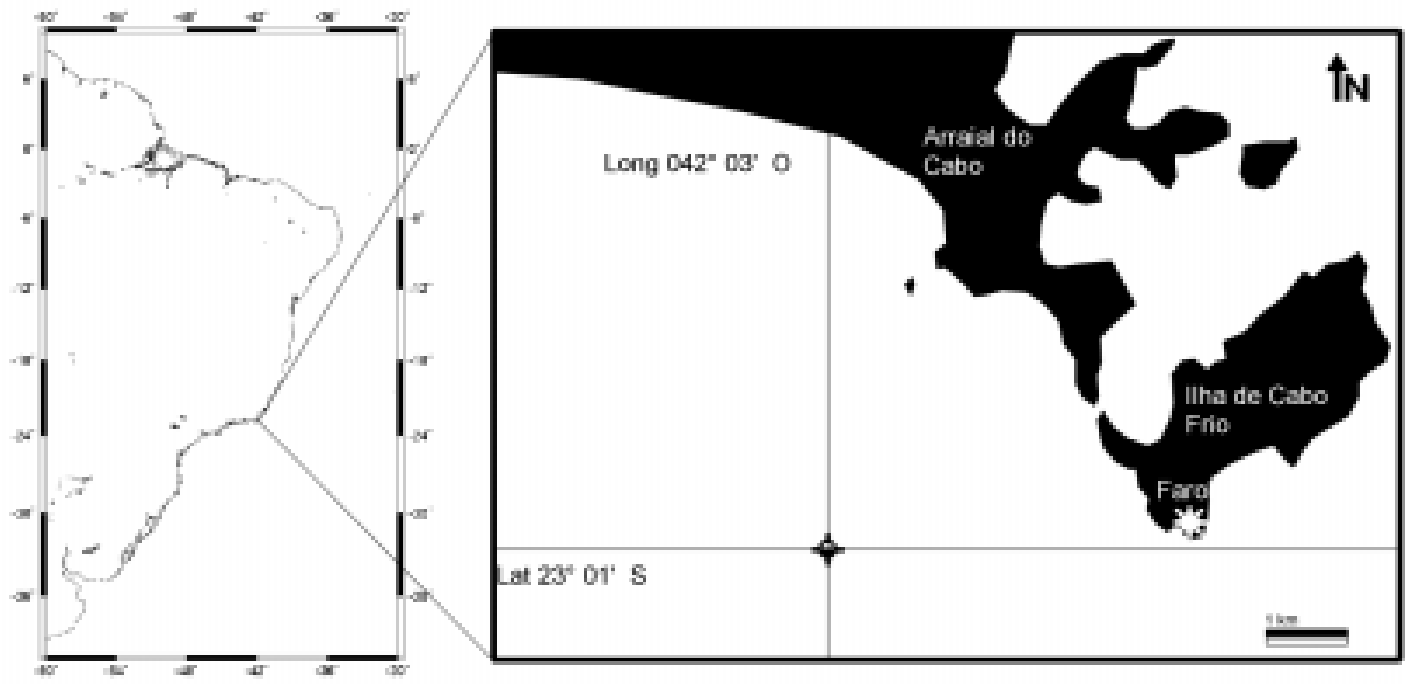

Figura 1 - Localização do ondógrafo. Note que o mesmo está localizado na zona de sombra das ondas de NE. 
lâmina d'água de 82 metros, distando de aproximadamente 3 milhas a oeste do Farol da llha de Cabo Frio.

\section{MATERIAIS E MÉTODOS}

O ondógrafo direcional utilizado foi do tipo WAVERIDER DATAWELL. O mesmo obtém informações instantâneas de acelerações nas direções vertical, E-O e N-S, integra duplamente com relação ao tempo para determinar o deslocamento nestas direções e envia o sinal em tempo real, via rádio $(27-40 \mathrm{MHz})$, para um receptor em terra.

As medições foram efetuadas a cada meia hora com taxa de amostragem de 1,28 $\mathrm{Hz}$ e duração de 20 minutos. Caso a altura significativa de ondas do registro fosse maior do que 2,0 metros o mesmo era armazenado automaticamente. Caso contrário, o registro de ondas somente era armazenado em intervalos de 3 horas. Foram produzidos um total de 7500 registros em toda a campanha.

As séries temporais foram submetidas inicialmente a testes de consistência para eliminação de dados espúrios que pudessem inserir erros na análise de curto termo. Os testes de consistência identificavam valores fora do limite de detecção do equipamento e "spikes". Se mais de 20 pontos defeituosos fossem identificados numa mesma série temporal esta era automaticamente descartada. Caso contrário, os valores eram substituídos pela média entre os pontos vizinhos. Do total, apenas $287(3,83 \%)$ arquivos foram corrigidos e $6(0,08 \%)$ foram reprovados.

Em seguida, as séries temporais consistidas foram submetidas a rotinas de cálculo do espectro direcional elaboradas em Matlab seguindo a formulação descrita abaixo:

Formulações espectrais direcionais para análise de curto termo

O espectro direcional de ondas, $\mathrm{S}(\mathrm{f}, \theta)$, pode ser estimado através de :

$$
S(f, \theta)=S(f) D(f, \theta)
$$

onde $S(f)$ é o espectro simples da série temporal da elevação da superfície e $D(f, \theta)$ é a Função de Espalhamento Angular (FEA). Kuik et al. (1988), propôs a modelagem da FEA em função de seus momentos angulares definindo uma direção principal $\theta$ e uma largura direcional $\sigma$ por intervalo de freqüência. Estes são determinados por:

$$
\begin{gathered}
\theta=\tan ^{-1}\left(\frac{b_{1}}{a_{1}}\right) \\
\sigma=\sqrt{2-2 \sqrt{a_{1}^{2}+b_{1}^{2}}}
\end{gathered}
$$

onde

$$
\begin{aligned}
& a_{1}=\frac{Q_{12}}{S_{11} k} \\
& b_{1}=\frac{Q_{13}}{S_{11} k}
\end{aligned}
$$

sendo $Q_{12}$ e $Q_{13}$, no caso do ondógrafo WAVERIDER, os espectros de quadratura entre as séries de deslocamento vertical e os deslocamentos nas direções E-O e N-S, respectivamente; $k$ é o número de onda por intervalo de freqüência definido por

$$
k=\sqrt{\frac{S_{22}+S_{33}}{S_{11}}}
$$

$S_{11}, S_{22}$ e $S_{33}$ são os auto-espectros dos deslocamentos vertical, E-O e N-S respectivamente.

Os espectros foram calculados aplicando-se Transformada Rápida de Fourier diretamente sobre séries temporais de 1.024 pontos com alisamento de 14 graus de liberdade. Da 
análise de curto termo foram extraídos parâmetros espectrais direcionais como altura significativa (HM0), período de pico (TP), direção principal (DIRTP) e espalhamento angu$\operatorname{lar}$ (ESPAL) associadas ao período de pico. As séries temporais dos parâmetros espectrais foram então reamostradas em intervalos horários e agrupadas em histogramas simples e de ocorrência conjunta de HMO x TP, HMO x DIRTP e TP x DIRTP, ESPAL X TP e ESPAL e DIRTP. Um total de 10.449 informações horárias foram utilizadas no processamento.

\section{RESULTADOS E DISCUSSÃO}

As Figuras 2-a a 2-d apresentam os histogramas simples das propriedades amostradas (HMO, TP, DIRTP e ESPAL). As alturas significativas das ondas medidas variaram entre 53 e $687 \mathrm{~cm}$ seguindo uma distribuição semelhante às funções Rayleigh e Lognormal, com média em $162 \mathrm{~cm}$, moda entre 130 e $140 \mathrm{~cm}$ e cerca de $63 \%$ das HM0 ocorrendo entre 80 e $180 \mathrm{~cm}$. Os períodos de pico apresentaram distribuição normal entre 3,9 e 20,0 segundos com moda entre 9 e 10 segundos e média de 10,3 segundos. Em $72 \%$ dos casos o período de pico variou entre 7 e 12 segundos. As ondas vieram predominantemente de SSE a SSO com $49,4 \%$ das mesmas variando entre 160 e 200 graus. O espalhamento angular seguiu uma distribuição quase normal, levemente assimétrica positivamente. O mesmo variou entre 8,5 e 69,8 graus com moda entre 25 e 30 graus.
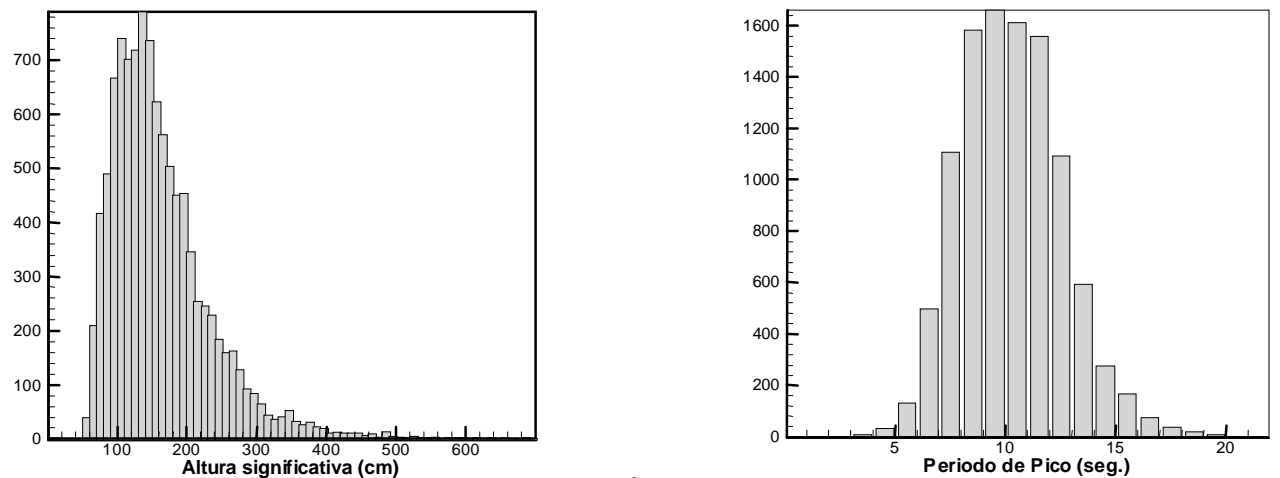

a)
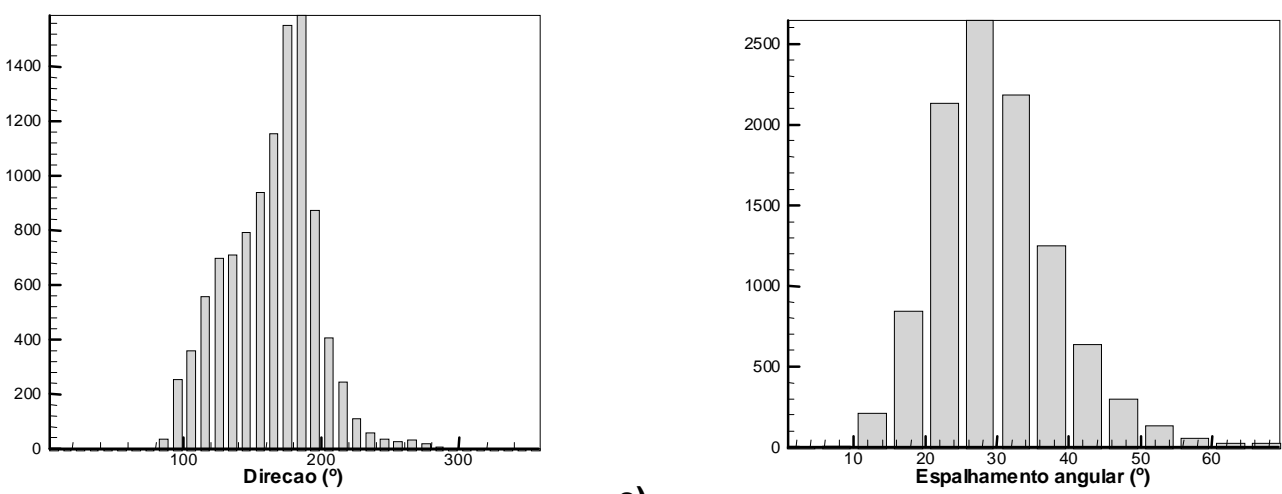

c)

Figura 2 - Histogramas simples para : a) Altura significativa (HM0); b) Período de Pico (TP); c) Direção principal (DIRTP) e d) Espalhamento angular (ESPAL). 
Cabe ressaltar que para a lâmina d'água de instalação do ondógrafo, somente ondas com períodos superiores a 10,3 segundos estariam efetivamente em águas profundas. Em $51 \%$ dos registros os períodos de pico estiveram acima deste valor.

A distribuição de ocorrência conjunta entre HMO e TP (Figura 3) mostra que as ondas com alturas significativas predominantes, entre 100 e $150 \mathrm{~cm}$, apresentam TP mais freqüente entre 8 e 10 segundos. Ondas com $\mathrm{HM0}$ maiores, superiores a $200 \mathrm{~cm}$, ocorreram predominantemente com períodos entre $10 \mathrm{e}$ 12 segundos, enquanto que as ondas com períodos maiores do que 12 segundos ocorreram mais freqüentemente com alturas menores do que $200 \mathrm{~cm}$.

A Figura 3 apresenta a ocorrência conjunta entre HS e DIRTP. Observa-se claramente a pouca freqüência de ondas vindas de SSO a O (210 a 290 graus). Este fato é, obviamente, causado pela pista reduzida que impede que ventos desse quadrante gerem ondas suficientemente grandes. Nota-se também que a maior parte da energia provem de SSE a S (160 a 190 graus), de ondas com alturas predominando entre 100 e $200 \mathrm{~cm}$. As ondas vindas de ESE possuem alturas predominando entre 100 e $130 \mathrm{~cm}$.

Observando-se a Figura 4, de ocorrência conjunta entre DIRTP e TP observa-se nitidamente a presença de dois picos dominantes: ondas vindas de ESE a SSE (110 a 140 graus), com períodos de pico entre 7 e 9 segundos; e ondas provenientes de SSE a SSO (160 a 200 graus) com períodos variando entre 10 e 13 segundos.

\section{Considerações sobre o espalhamento angular}

A Figura 6 apresenta a distribuição conjunta do espalhamento pela direção do período de pico. Pode-se observar um pico dominante entre as direções 170 e 190 graus com espalhamento 25 a 35 graus. Observa-se ainda uma incidência acentuada de ondas variando de sul para sudeste com espalhamento de- crescente, concentrando em 20 e 25 graus entre os azimutes 120 e 130 graus.

A Figura 7 apresenta o espalhamento por faixa de período de pico. Os estados de mar com períodos de pico entre 9 e 12 segundos, típicos das ondulações de Sul, apresentaram espalhamento predominantemente entre 25 e 35 graus, enquanto que os de TP entre 8 e 10 segundos, característicos das ondas de Sudeste, apresentaram espalhamento entre 20 e 25 graus.

Este quadro sugere que as ondas de SE possuem espalhamento menor do que as ondas de sul. Tal suposição parece contraditória uma vez que ondas de sul estariam mais afastadas da zona de geração, e assim mais selecionadas do que as de sudeste, sugerindo um menor espalhamento.

Seixas, 1997, numa tentativa de parametrizar parâmetros direcionais de onda para a Bacia de Campos, apresenta valores de espalhamento angular para os primeiros, segundos e terceiros picos de energia, assim selecionados por ordem de tamanho, que ocorressem no espectro. Os primeiros picos apresentaram espalhamento predominante de 20 graus enquanto que nos demais picos 0 espalhamento mais freqüente foi 25 graus. Infelizmente, nos histogramas apresentados pelo autor, não há como saber em que faixas de freqüência ocorreram esses picos. Entretanto, a mesma metodologia, quando aplicada aos períodos de pico revela que os primeiros picos ocorrem preferencialmente na faixa de 8 e 9 segundos. Tais períodos são característicos de ondas provenientes de SE, o que vem a reforçar o fato de que as ondas de SE realmente possuem menor espalhamento.

Há uma certa dificuldade em amostrarse corretamente o espalhamento angular, que pela sua natureza de cálculo pode muito sensível a ruídos, principalmente com sensores tipo "pitch-and-roll" (Steele ,et al., 1985). A correta estimativa do mesmo requer a aplicação de funções de transferência para correção dos efeitos que o fundeio exerce sobre a bóia e da inércia da própria bóia que tenta reproduzir satisfa- 
toriamente a inclinação da superfície do mar. uma vez que o que a bóia mede efetivamente é No caso do ondógrafo direcional WAVERIDER o seu deslocamento horizontal e vertical, tenesse problema parece ter menor importância tando reproduzir os movimentos das partículas

\begin{tabular}{|c|c|c|c|c|c|c|c|c|c|c|c|c|c|c|c|c|c|c|c|c|}
\hline HMO (cm) & í & $\begin{array}{l}n \\
: \\
\dot{q}\end{array}$ & $\begin{array}{l}0 \\
\dot{1} \\
\end{array}$ & $\hat{0}$ & $\begin{array}{l}\infty \\
1 \\
1\end{array}$ & $\begin{array}{l}0 \\
1 \\
\infty\end{array}$ & 우 & $\begin{array}{l}\bar{T} \\
\grave{\Gamma}\end{array}$ & $\begin{array}{l}\stackrel{N}{T} \\
\stackrel{+}{T}\end{array}$ & $\begin{array}{l}\stackrel{m}{+} \\
\stackrel{\sim}{\sim}\end{array}$ & 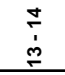 & $\begin{array}{l}\stackrel{6}{2} \\
\dot{5} \\
\frac{1}{1}\end{array}$ & 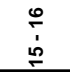 & 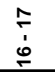 & $\begin{array}{l}\infty \\
\\
\end{array}$ & $\begin{array}{l}\stackrel{0}{T} \\
\vdots \\
\infty \\
\end{array}$ & 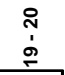 & total & Tp médio & $\%$ \\
\hline $50-60$ & & & & 1 & 7 & & 2 & 4 & 11 & 13 & 1 & & & & & & & 39 & 10.9 & 0.37 \\
\hline $60-70$ & & & & 1 & -31 & 20 & 17 & 29 & 45 & 32 & 22 & 5 & 7 & & & & & 209 & 10.9 & 2.00 \\
\hline $70-80$ & & & 2 & 19 & 79 & 66 & 48 & 51 & 50 & 27 & 37 & 10 & 17 & 8 & 1 & 1 & & 416 & 10.3 & 3.98 \\
\hline $80-90$ & 5 & 4 & 12 & 17) & 102 & 102 & 55 & 68 & 49 & 29 & 12 & 18 & 10 & 3 & 1 & & 2 & 489 & 9.6 & 4.68 \\
\hline $90-100$ & 1 & 1 & 4 & 50 & 110 & 129 & 720 & 74 & 81 & $3 \lambda$ & 19 & 24 & 10 & 3 & 2 & 1 & 1 & 667 & 9.7 & 6.38 \\
\hline $100-110$ & & 9 & 2 & 59 & 27 & 146 & 125 & 109 & 70 & 50 & 31 & 15 & 6 & 4 & 11 & 5 & 1 & 740 & 9.8 & 7.08 \\
\hline $110-120$ & 2 & 3 & 11 & 49> & 88 & 161 & 185 & J10 & 54 & 48 & 18 & 5 & 10 & 5 & 2 & 3 & 2 & 702 & 9.6 & 6.72 \\
\hline $120-130$ & & 1 & 14 & 5. & $1 k$ & 140 & $\pi 11$ & 116 & 62 & 53 & 30 & 4 & 10 & 6 & 1 & 1 & & 718 & 9.6 & 6.87 \\
\hline $130-140$ & & 4 & 18 & 71 & 89 & 134 & 123 & 109 & 99 & 65 & 29 & 26 & 14 & 5 & 2 & 1 & & 789 & 9.9 & 7.55 \\
\hline $140-150$ & & 5 & 15 & 43 & 79 & 174 & 133 & -124 & 111 & 68 & 22 & 3 & 11 & 2 & 4 & 2 & & 736 & 9.9 & 7.04 \\
\hline $150-160$ & & 3 & 9 & 31 & 67 & 91 & 108 & 78 & 94 & >85 & 28 & 8 & 7 & 8 & 5 & 1 & & 623 & 10.2 & 5.96 \\
\hline $160-170$ & & 2 & 7 & 21 & $5 \lambda$ & 83 & 99 & 82 & 83 & 56 & 37 & 19 & 9 & 8 & 5 & 2 & & 564 & 10.5 & 5.40 \\
\hline $170-180$ & & & 11 & 11 & 21 & 66 & 84 & 96 & शे & 49 & 33. & 26 & 6 & 5 & 1 & & & 504 & 10.7 & 4.82 \\
\hline $180-190$ & & 1 & 8 & 10 & 21) & 49 & 68 & 95 & 70 & 62 & 29 & 18 & 12 & 8 & & & & 451 & 10.8 & 4.32 \\
\hline $190-200$ & & & 7 & 16 & 26 & 73 & 60 & -63 & 72 & 77) & 34 & 17 & 9 & & & & & 454 & 10.6 & 4.34 \\
\hline $200-210$ & & & 4 & 8 & 9 & 42 & 58 & 54 & 64 & 54 & 33 & 12 & 5 & 4 & & & & 347 & 10.9 & 3.32 \\
\hline $210-220$ & & & 1 & 9 & 18 & 25 & 34 & 42 & 57 & 34 & 19 & 8 & 4 & 2 & & & & 253 & 10.8 & 2.42 \\
\hline $220-230$ & & & 2 & 7 & 20 & 26 & 35 & 47 & 51 & 38 & 13 & 4 & 2 & & & & & 245 & 10.5 & 2.34 \\
\hline $230-240$ & & & & 8 & 18 & 22 & 30 & 42 & 43 & 40 & 20 & 4 & 1 & & & & & 228 & 10.7 & 2.18 \\
\hline $240-250$ & & & & 6 & 15 & 12 & 29 & 37 & 39 & 23 & 17 & 4 & 2 & & & & & 184 & 10.8 & 1.76 \\
\hline $250-260$ & & & 1 & 5 & 15 & 24 & 34 & 34 & 26 & 9 & 9 & 1 & & 2 & & & & 160 & 10.2 & 1.53 \\
\hline $260-270$ & & & & 3 & 12 & 18 & 26 & 27 & 36 & 22 & 13 & 4 & 1 & & & 1 & & 163 & 10.7 & 1.56 \\
\hline $270-280$ & & & 1 & & 7 & 16 & 20 & 21 & 36 & 11 & 9 & 1 & 5 & & 1 & & & 128 & 10.8 & 1.22 \\
\hline $280-290$ & & & & 1 & 4 & 9 & 18 & 20 & 19 & 12 & 2 & 5 & 3 & & & & & 93 & 10.9 & 0.89 \\
\hline $290-300$ & & & & 1 & 4 & 9 & 14 & 6 & 20 & 18 & 7 & 2 & 2 & 1 & & & & 84 & 11.1 & 0.80 \\
\hline $300-310$ & & & & & 3 & 4 & 11 & 8 & 16 & 9 & 6 & 7 & 1 & & & & & 65 & 11.4 & 0.62 \\
\hline $310-320$ & & & & & 1 & 1 & 5 & 10 & 10 & 7 & 7 & 2 & & & & & & 43 & 11.5 & 0.41 \\
\hline $320-330$ & & & & & & 1 & 2 & 7 & 7 & 11 & 7 & & & & & & & 35 & 11.8 & 0.33 \\
\hline $330-340$ & & & & & 1 & 2 & 7 & 5 & 9 & 10 & 5 & 1 & & 1 & & & & 41 & 11.5 & 0.39 \\
\hline $340-350$ & & & & & & 1 & 8 & 13 & 16 & 8 & 4 & 1 & & & & & & 51 & 11 & 0.49 \\
\hline $350-360$ & & & & & & & 1 & 9 & 10 & 7 & 4 & 1 & & & & & & 32 & 11.7 & 0.31 \\
\hline $360-370$ & & & & & & 1 & 2 & 6 & 9 & 5 & 3 & & & & & & & 26 & 11.5 & 0.25 \\
\hline $370-380$ & & & & & & & 6 & 2 & 13 & 7 & 1 & & & & & & & 29 & 11.3 & 0.28 \\
\hline $380-390$ & & & & & & 1 & 1 & 6 & 9 & & 3 & 1 & & & & & & 21 & 11.5 & 0.20 \\
\hline $390-400$ & & & & & & 2 & 3 & 4 & 3 & 2 & 3 & 1 & & & & & & 18 & 11.2 & 0.17 \\
\hline $400-410$ & & & & & & & 1 & 2 & 6 & & & & & & & & & 9 & 10.9 & 0.09 \\
\hline $410-420$ & & & & & & & & 2 & 3 & 3 & 1 & 2 & & & & & & 11 & 12.4 & 0.11 \\
\hline $420-430$ & & & & & & 1 & 1 & 1 & 2 & 1 & 1 & 3 & & & & & & 10 & 12.0 & 0.10 \\
\hline $430-440$ & & & & & & 1 & 1 & 3 & 1 & 1 & 3 & & & & & & & 10 & 11.6 & 0.10 \\
\hline $440-450$ & & & & & & 1 & & 3 & 1 & 2 & 2 & 1 & & & & & & 10 & 12.0 & 0.10 \\
\hline $450-460$ & & & & & & & 2 & & & 1 & 2 & 1 & & & & & & 6 & 12.3 & 0.06 \\
\hline $460-470$ & & & & & & & 1 & 1 & 4 & & & 2 & & & & & & 8 & 11 & 0.08 \\
\hline $470-480$ & & & & & & & & & 1 & 1 & 1 & & & & & & & 3 & 12.2 & 0.03 \\
\hline $480-490$ & & & & & & & 1 & & 2 & 1 & 3 & 4 & 1 & & & & & 12 & 13 & 0.11 \\
\hline $490-500$ & & & & & & & & 1 & & & 1 & 2 & & & & & & 4 & 13.5 & 0.04 \\
\hline $500-510$ & & & & & & & & 1 & & & 1 & & & & & & & 2 & 12.3 & 0.02 \\
\hline $510-520$ & & & & & & & & & & & & 1 & & & & & & 1 & 14.6 & 0.01 \\
\hline $520-530$ & & & & & & & & & 1 & 1 & & 2 & & & & & & 4 & 13.4 & 0.04 \\
\hline $530-540$ & & & & & & & & & & & 1 & & & & & & & 1 & 13.3 & 0.01 \\
\hline $540-550$ & & & & & & & & & & & 1 & & & & & & & 1 & 13.3 & 0.01 \\
\hline $550-560$ & & & & & & & & & & 1 & 1 & 1 & & & & & & 3 & 13.6 & 0.03 \\
\hline $560-570$ & & & & & & & & & & & & & & & & & & 0 & & 0.00 \\
\hline $570-580$ & & & & & & & & & & & 1 & & & & & & & 1 & 13.3 & 0.01 \\
\hline $580-590$ & & & & & & & & & & & 1 & & & & & & & 1 & 13.7 & 0.01 \\
\hline $590-600$ & & & & & & & & & & & 1 & & & & & & & 1 & 13.7 & 0.01 \\
\hline $600-610$ & & & & & & & & & & & & & & & & & & 0 & & 0.00 \\
\hline $610-620$ & & & & & & & & & & & 1 & & & & & & & 1 & 13.3 & 0.01 \\
\hline $620-630$ & & & & & & & & & & & & & & & & & & 0 & & 0.00 \\
\hline $630-640$ & & & & & & & & & & & 1 & & & & & & & 1 & 13.6 & 0.01 \\
\hline $640-650$ & & & & & & & & & & & & & & & & & & 0 & & 0.00 \\
\hline $650-660$ & & & & & & & & & & & & & 1 & & & & & 1 & 15.4 & 0.01 \\
\hline $660-670$ & & & & & & & & & & & & & & & & & & 0 & & 0.00 \\
\hline $670-680$ & & & & & & & & & & & & & & & & & & 0 & & 0.00 \\
\hline $680-690$ & & & & & & & & & & & 1 & & & & & & & 1 & 13.9 & 0.01 \\
\hline $\begin{array}{r}\text { total } \\
\text { IMO médio }\end{array}$ & $\begin{array}{c}8 \\
95\end{array}$ & $\begin{array}{c}33 \\
124.2\end{array}$ & $\begin{array}{c}129 \\
145.6\end{array}$ & $\begin{array}{l}498 \\
137.3\end{array}$ & $\begin{array}{l}1108 \\
133.9\end{array}$ & $\begin{array}{l}1583 \\
145.1\end{array}$ & $\begin{array}{l}1659 \\
159.6\end{array}$ & $\begin{array}{l}1613 \\
168.1\end{array}$ & $\begin{array}{l}1560 \\
180\end{array}$ & $\begin{array}{l}1090 \\
178.5\end{array}$ & $\begin{array}{c}591 \\
189.6\end{array}$ & $\begin{array}{c}276 \\
185.8\end{array}$ & $\begin{array}{c}166 \\
152.3\end{array}$ & $\begin{array}{c}75 \\
149\end{array}$ & $\begin{array}{c}36 \\
132.8\end{array}$ & $\begin{array}{c}18 \\
130.1\end{array}$ & $\begin{array}{c}6 \\
100.8\end{array}$ & 10449 & & 100 \\
\hline Miv inecuso & 0.08 & 0.32 & $\begin{array}{l}1.23 \\
1.23\end{array}$ & 4.77 & 10.60 & 15.15 & 15.88 & 15.44 & $\begin{array}{l}100 \\
14.93\end{array}$ & 10.43 & $\begin{array}{c}109.0 \\
5.66\end{array}$ & 2.64 & 1.59 & 0.72 & 0.34 & 0.17 & 0.06 & 100 & & \\
\hline
\end{tabular}

Figura 3 - Diagrama de ocorrência conjunta de Altura significativa e Período de pico (HM0 x TP). 
de água. Não havendo influência significativa do fundeio sobre o equipamento, não há porque a medida não ser confiável. Sugere-se, portanto, que a estimativa do espalhamento feita pelo ondógrafo seja coerente e que o espalhamento angular diferenciado entre as ondas de SE e S sejam resultado das características climáticas de suas zonas de geração. Caberá, portanto, numa fase posterior, uma análise mais profunda das cartas sinópticas procurando observar a variabilidade temporal e espacial dos ventos dentro das zonas de geração de cada um destes estados de mar.

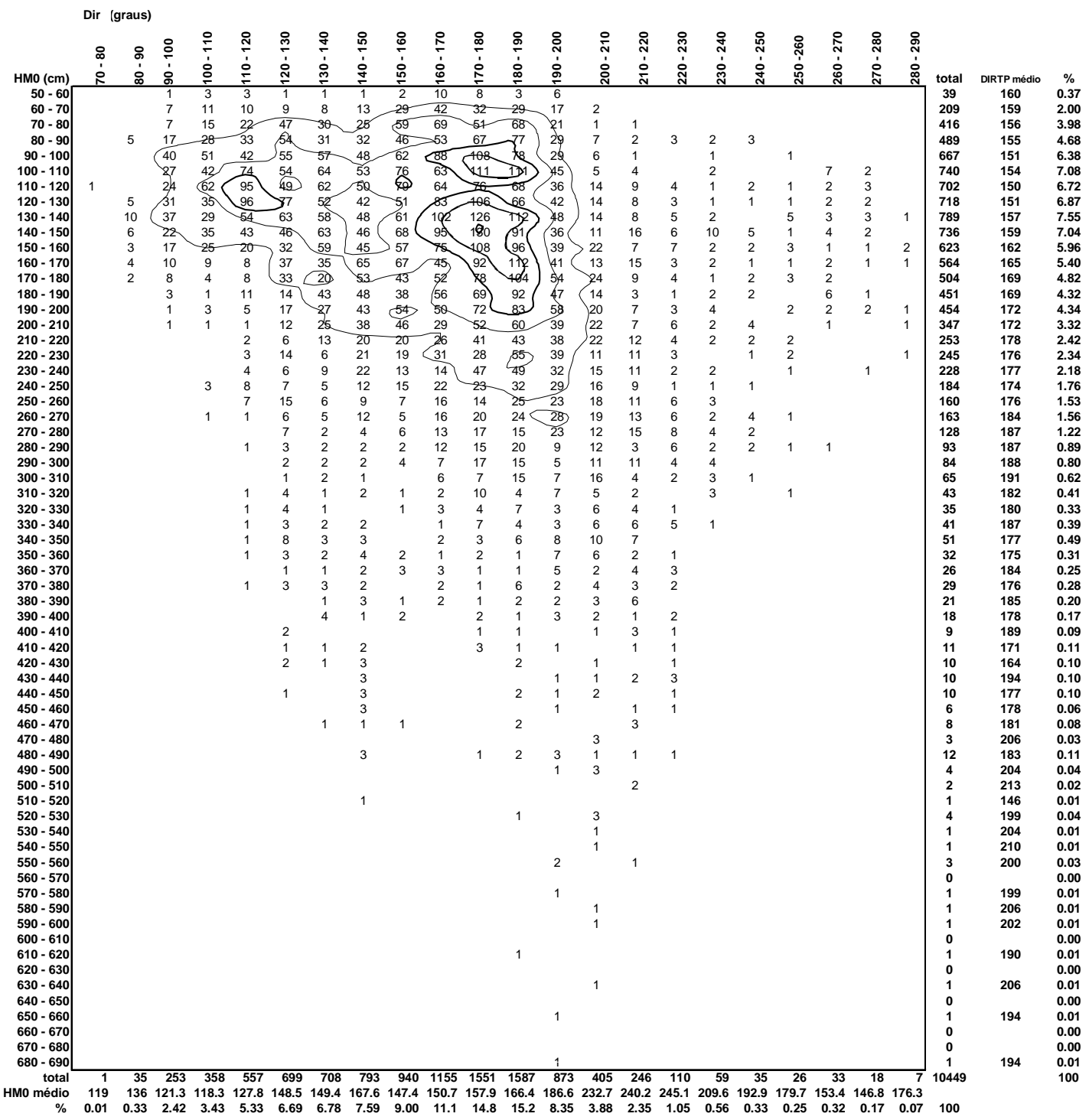

Figura 4 - Diagrama de ocorrência conjunta de Altura significativa e Direção principal (HM0 x DIRTP). 
Dir (graus)

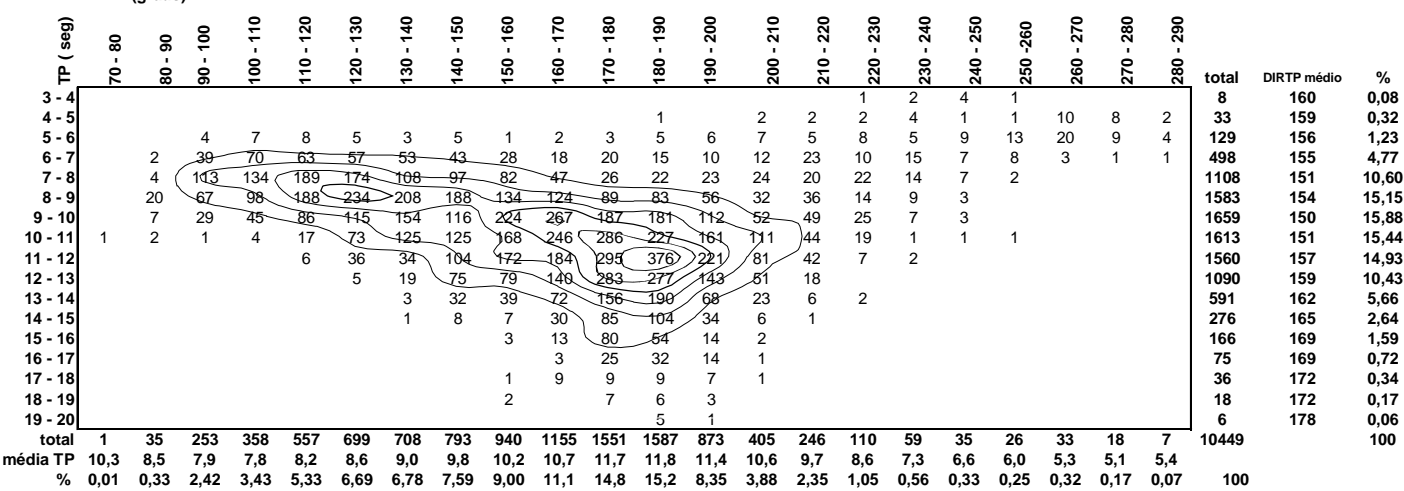

Figura 5 - Diagrama de ocorrência conjunta de Período de pico e Direção principal (TP x DIRTP).

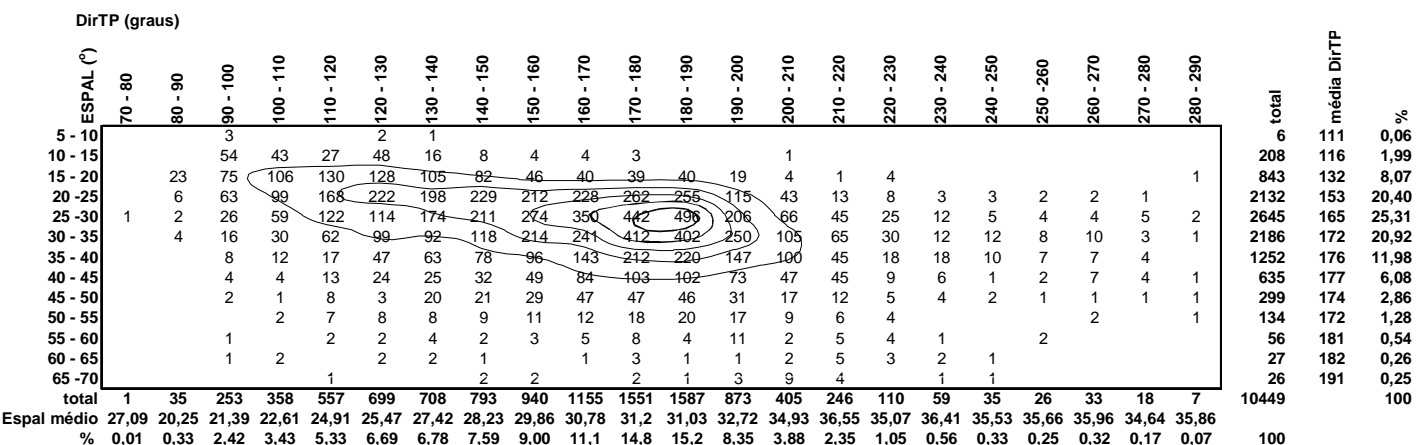

Figura 6 - Diagrama de ocorrência conjunta de Espalhamento angular e Direção principal (ESPAL x DIRTP).

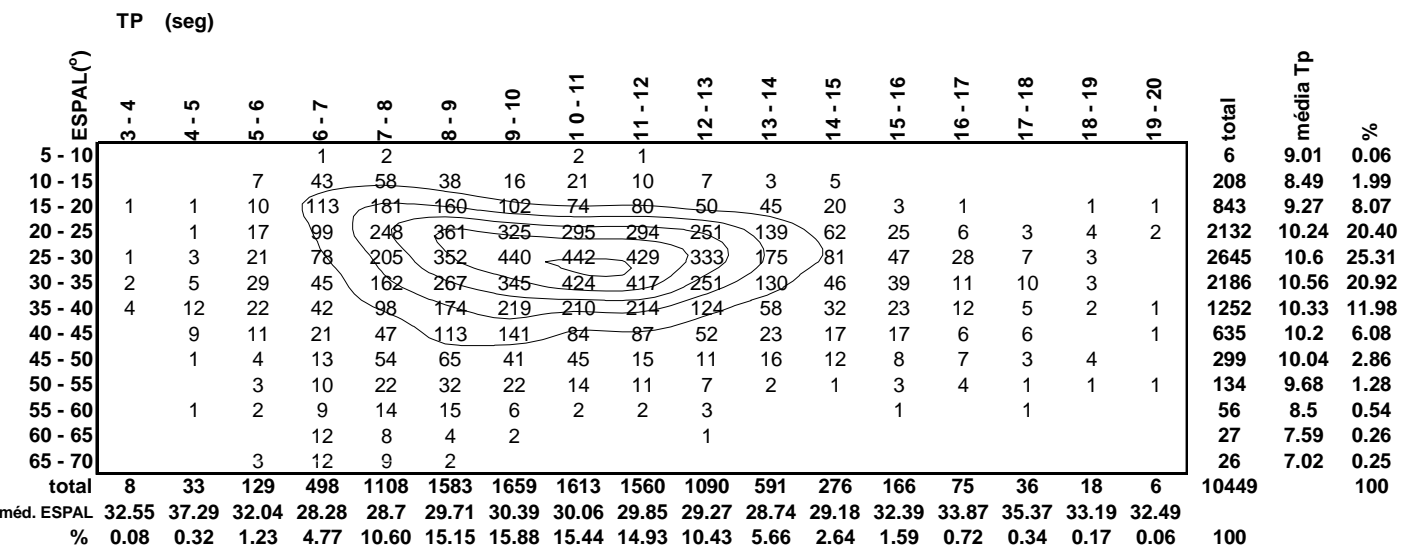

Figura 7 - Diagrama de ocorrência conjunta de Espalhamento angular e Período de Pico (ESPAL x TP). 


\section{CONCLUSÕES}

Analisou-se um ano de informações de onda direcional obtidos na região próxima à costa sul de Arraial do Cabo. A maior parte da energia provêm de ondas vindas de SSE a S, com alturas significativas entre 100 e $200 \mathrm{~cm}$ e períodos variando entre 11 e 13 segundos. Um segundo pico de energia vem de ESE a SSE com períodos predominando entre 7 e 9 segundos.

O espalhamento angular para ondas vindas de SE são menores do que o das de Sul. Tal fato pode estar relacionado a característica do campo de ventos nas zonas de geração de cada ondulação.

\section{AGRADECIMENTOS}

Os autores sinceramente agradecem ao Instituto de Estudos do Mar Almirante Paulo Moreira pela realização impecável da campanha de medição de ondas e pelas facilidades oferecidas que permitiram a permanência da primeira autora em suas instalações. Agradecem também à Pro-Reitoria de Ensino, Pesquisa e extensão da Universidade do vale do Itajaí pela liberação de recursos para a bolsa de estudos do artigo 170 e despesas de custeio do projeto.

\section{REFERÊNCIAS BIBLIOGRÁFICAS}

Kuik, A.J.; van Vledder \& L.H. Holthuijsen, 1988. A Method for the Routine Analysis of Pitchand-Roll Buoy Wave Data. Journal of Physical Oceanography. 18: 1020-1034.

Seixas, A.B.S., 1997. Clima de Ondas na Bacia de Campos: Análise dos Dados e Proposta de Parametrização. Tese de Doutorado, Observatório Nacional.

Steele, K.E.; Lau, J.C.K. \& Y.H.L. Hsu, 1985. Theory and Application of Calibration Techniques for na NDBC Directional Wave Measurements Buoy. IEEE, Journal of Oceanic Engineering. OE-10(4):382-396.
Strauch, J.C. \& R.M. Schimidt, 1998. Um Ano de Monitoramento de Ondas em Rio Grande. Resumos Expandidos da XI Semana Nacional de Oceanografia, pp. 357-359. Rio Grande, RS.

Violante Carvalho, N.; Nunes, L.M.P. \& W. Tavares Jr., 1995. O Clima de Ondas da Bacia de Campos. Anais do XII Simpósio Brasileiro de Recursos Hídricos. Vitória, ES.

Violante Carvalho, N. 1998. Investigação sobre a Evolução do Clima de Ondas na Bacia de Campos e a Correlação com as Situações Meteorológicas Associadas. Tese de mestrado, Universidade Federal do Rio de Janeiro. 